\title{
Prozeßleitsysteme mit Rechnernetz für die Überwachung, Steuerung und Berechnung thermischer Trennprozesse, Apparate und Anlagen
}

\author{
Prof. Dr-Ing. Josef Schmadl
}

\begin{abstract}
An der Technischen Fachhochschule Wildau wurde ein Verbund Prozeßleitsystem-Rechnernetz entwickelt und in Kooperation mit der Firma Hartmann \& Braun, Frankfurt realisiert und in Betrieb genommen. Das Verbundsystem wird sowohl für die Berechnung thermischer Trennprozesse (stationäre Simulation) und für die direkte Steuerung der Apparaturen im Technikum für thermische Verfahrenstechnik verwendet. Dabei ist ein On-line-Datenaustausch zwischen Rechnerteil und Prozeßleitsystem möglich.
\end{abstract}

\section{Beschreibung der Hardwarekonfiguration}

Als Hardware steht ein Rechnernetz, das mit einem Prozeßleitsystem Marke CONTRONIC P der Firma Hartmann \& Braun und nötigen Peripheriegeräten verknüpft ist, zur Verfügung. Das Prozeßleitsystem dient als zentrale MSREinheit für ein Technikum für Thermische Verfahrenstechnik und ermöglicht u. a. sicherheitstechnische Überwachung und MSR-technische Steuerung sowie auch direkte Kopplung des Rechnernetzes mit verfahrenstechnischen Apparaturen (Anlage 1).

Das Rechnernetz wird in den Lehrfächern Thermodynamik, Wärme- und Stoffübertragung und Thermische Verfahrenstechnik sowie im Rahmen von Forschungsvorhaben genutzt. Es besteht im wesentlichen aus einer UNIXWorkstation, verknüpft über Ethernet mit fünf 486'er DOS-PC's. Es ist mit einem Software-Paket ausgestattet, das die Bearbeitung von Echtzeit-Fragestellungen für Zwecke der dynamischen Simulation und Prozeßsteuerung ermöglicht. Es ist auf der einen Seite mit Simulationssoftware und auf der anderen Seite über das Prozeßleitsystem mit verfahrenstechnischen Prozessen koppelbar. Auf dem Rechnernetz sind sowohl UNIX-Anwendungen wie auch DOS/WINDOWS-Anwendungen lauffähig. Das gesamte System ist in Betrieb genommen und getestet. Ebenfalls bereits vorhanden und installiert sind die Simulations-Softwareprogramme ASPEN PLUS (als UNIX-Anwendung) und CHEMCAD (als DOS-Anwendung) in jeweils neuester Version. Die Hardwarekonfiguration des gesamten Verbundsystems mit Peripheriegeräten ist in dem anliegenden Kabelplan dargestellt (Anlage 2).

\section{Beschreibung der Einzelsysteme}

\subsection{Prozeßleitsystem}

a) Gruppenleitstation (GLS 001)

An die Gruppenleitstation ist ein Bedienplatz, bestehend aus insgesamt einem Monitor, einer Bedientastatur, ei- nem Lichtgriffel und einem Drucker angeschlossen. Hauptfunktionen der Gruppenleitstation sind:

- Prozeßvisualisierung, z. B. mit Hilfe der Übersichtsbilder, Einblendebilder, Kurvenbilder,

- sicherheitstechnische Prozeßüberwachung, z. B. mit Hilfe der Störmeldungen,

- Prozeßsteuerung über die Prozeßstationen (PS 030). Sowohl die standardmäßig konfektionierten Bilder wie die Übersichtsbilder, Gruppenbilder, Alarmseiten und Kurvendarstellungen als auch das anwenderspezifisch zu erzeugende Fließbild mit lebenden Meßwerten können wahlweise auf dem Monitor aufgeschaltet werden. Das Fließbild kann auf dem Monitor gerollt werden. Die Bedienstation hat Zugriff auf einen integrierten 80 MByteFestplattenspeicher. Der angeschlossene Drucker ist für spontane und zyklische Protokolle sowie für Ausdrucke der Bildschirminhalte vorgesehen.

\section{b) Prozeßstation (PS 030)}

Zur Prozeßstation sowie zur Realisierung der Automatisierungsfunktion Regeln, Steuern und Überwachen dienen die Prozeßstationen, die in einem Systemschrank PS 030 untergebracht sind. Die Prozeßinterface-Komponenten zur Signalaufbereitung aus dem Prozeß sind Bestandteile der Prozeßstationen. Die Speisespannungsversorgung ist bei allen Prozeßinterface-Komponenten vorhanden. Alle Prozeßsignaleingänge und -ausgänge des Contronic-P-Leitsystems werden in den ProzeßinterfaceKomponenten von der Versorgungsspannung und dem Systembus galvanisch getrennt. Ex-Trennstellen sind ohne zusätzlichen Platzbedarf in das Contronic-P-System integriert. Im einzelnen sind folgende ProzeßinterfaceKomponenten vorhanden:

5 Stück Analogeingabe PEA 61-Ex/4 Eingänge à $4 \ldots 20 \mathrm{~mA}$ 7 Stück Analogeingabe PEA 62-Ex/8 Eingänge à $0 / 4 \ldots 20 \mathrm{~mA}$ 20 Stiuck Reglerstellausgabe PAR 61-Ex/1-kanalig à $0 / 4 \ldots 20 \mathrm{~mA}$ 3 Stück Binäreingabe PEB 63-Ex/30-kanalig $14 \mathrm{~V}$ 4 Stück Binärausgabe PAB 02/15 Ausgänge $19.5 \ldots 60 \mathrm{~V}$

\section{Übersichtsbilder/Gruppenbilder/Grafikbilder}

Vorgesehen ist eine hierarchisch gestufte Anwahl der Meßstellen mit konfektionierter Übersichtsdarstellung, bestehend aus Übersichtsbild (144 Meßstellen) und Gruppenbild (6 Meßstellen).

Die Anwahl kann entweder durch die Koordinatentastatur, den Lichtgriffel oder die Cursorsteuerung direkt aus dem Übersichts- bzw. Grafikbild erfolgen.

\section{Einblendbilder}

Die Einblendbildtechnik ermöglicht die gleichzeitige Darstellung der Übersichts- und Detaildarstellung. An- 
wenderspezifisch können so Einblendbilder mit beliebigen konfektionierten und freikonstruierten Bildern kombiniert werden. Das Einblendbild hat Vorrang vor den Informationen des „Hintergrundbildes“. Das Aufrufen mehrerer Einblendbilder unterschiedlichen oder gleichen Types in dasselbe Hintergrundbild ist bei gleichen Zeichensätzen möglich. Mögliche Einblendbildtypen:

- Loop-Einblendbild (z. B. Regelkreis)

(1/6 Bild im Hochformat, 28 Zeilen x 12 Spalten)

Das Loop-Einblendbild ist ein konfektioniertes Bild.

Es zeigt eine MSR-Stelle, wobei alle Darstellungs- und Bedienelemente der Darstellung im Gruppenbild entsprechen.

- Kurven-Einblendbild (z. B. Plot-Meßwert) (1/4 Bild im Querformat, 14 Zeilen x 40 Spalten) Das Kurven-Einblendbild ist ebenfalls ein konfektioniertes Bild. Es gibt den zeitlichen Verlauf von Prozeßgrößen wieder. Bis zu 3 Prozeßgrößen einer MSR-Stelle können im Kurven-Einblendbild dargestellt werden.

- Grafik-Einblendbild (z. B. Apparat/Verfahrensabschnitt) (1/4 Bild im Querformat, 14 Zeilen x 40 Spalten) Grafik-Einblendbilder sind frei konstruierbar. Sofern sie keine Kurvendarstellungen enthalten, können Grafik-Einblendbilder unabhängig vom Hintergrundbild eingeblendet werden. Die Darstellungs- und Bedienmöglichkeiten entsprechen den Grafikbildern.

\section{Kurvendarstellung/Grafikbilder (Fließbilder)/Archivierung als Standardfunktion}

Der zeitliche Verlauf von Meßgrößen wird mit Hilfe der Gruppenleitstationen erfaßt, abgespeichert und bei Bedarf dargestellt oder auf einen Archiv-Datenträger (Kassette oder Diskette) kopiert.

\section{- Kurvenbilder}

Für ständig zu verfolgende Größen werden Kurvenbilder konfiguriert, wobei die Meßwertverläufe in einer festen Gruppierung von max. 6 Meßstellen je Bild gemeinsam als Kurven dargestellt werden. Die Darstellung der Kurve wird aus 240 Bildpunkten auf der Zeitachse gebildet.

Zeitmaßstab: $1 \mathrm{~min} .4 \mathrm{~min} .20 \mathrm{~min} .2 \mathrm{~h} 8 \mathrm{~h} \quad 24 \mathrm{~h}$ Auflösung: $0.25 \mathrm{~s} \quad 1 \mathrm{~s} \quad 5 \mathrm{~s} \quad 30 \mathrm{~s} 2 \mathrm{~min} .6 \mathrm{~min}$.

- Variable Kurvenbilder

Nur vorübergehend interessante Meßwertverläufe, $z$. B. während einer Stör- und Anfahrphase, können im laufenden Betrieb zu variablen Kurvenbildern zusammengestellt werden. Je Leitstation sind 6 Variable zu je $6 \mathrm{Meßstellen} \mathrm{frei} \mathrm{belegbar.} \mathrm{Die} \mathrm{Auflösung} \mathrm{ist} \mathrm{mit} \mathrm{der}$ bereits vorher beschriebenen indentisch. Die Speicherung der Kurvendaten erfolgt in einem der Leitstation zugeordneten Festplattenspeicher. In diesem Massenspeicher können z. B. die Daten für 120 Kurven mit einer Auflösung von $30 \mathrm{~s}$ über eine Woche gespeichert werden.

- Grafikbilder

Grafikbilder sind für die jeweils benötigte Prozeßdarstellung in Einzelbildern frei konstruierbar. Maximal 56 Einzelbilder lassen sich zu einem Rollbild zusammenfassen. In jedem Grafikbild können analoge, binäre und digitale variable Größen dargestellt werden. Zur Speicherung der Grafikbilder steht der Massenspeicher ebenfalls zur Verfügung. Je Bild werden ca. 2 Kbyte auf dem Massenspeicher belegt.

\section{Störmeldungen}

Prozeßstörungen, die von der Prozeßstation erfaßt werden, sind unabhängig vom Bildtyp am oberen Bildrand auf dem Monitor in einem dafür reservierten Feld dargestellt. Es können die ersten 6 Meldungen gleichzeitig in Form des MSR-Namens mit zugehörigem Status angezeigt werden. Sind weitere Störmeldungen vorhanden, dann wird dieses durch eine Überlaufanzeige gekennzeichnet. Nach Betätigung der Alarmtaste werden alle Prozeßstörungen zeitfolgerichtig auf dem Monitor auf der „Alarmseite“ dargestellt.

Die Informationen werden auf mehreren „Alarmseiten“ geschrieben. 20 Seiten je 13 Meldungen mit Uhrzeit, MSR-Stelle, Kurztext und Alarmstatus sind standardmäßig möglich. Die kleinstmögliche zeitliche Auflösung zwischen den Störmeldungen beträgt $10 \mathrm{msec}$. Der Scanzyklus für die Alarmdarstellung beträgt $250 \mathrm{~ms}$. Jede Alarmstufe kann in 4 Prioritätsstufen (farbliche Unterscheidung) und 16 Anlagenbereiche während des Konfigurierens der MSR-Stelle eingordnet werden. Je Meßwerterfassung sind von 8 möglichen Grenzwerten 4 Grenzwerte zur Überwachung konfigurierbar. Selbstdiagnose und Fehlermeldung innerhalb des Contronic P Leitsystems erfolgen konfigurierbar oder als standardmäßig systemeigene Funktion.

Konfigurierbar ist die Überwachung auf:

- Gradienten

- Bereichsgrenzen

- Plausibilität

Wird bei diesen Prüfungen ein Fehler festgestellt, so wird Ereignismeldung auf dem Drucker angegeben und auf dem Monitor angezeigt.

Falls es konfiguriert ist, erfolgt:

- Umschalten auf Ersatzwert; Ersatzwerte können dynamisch von einer anderen Meßstelle kommen

- Zwangshand

- Sicherheitslage

- statische Ersatzwerte

- „Halten“ letzter Wert

Standardmäßige systemeigene Überwachung erfolgt für:

- Erdschluß (Kurzschluß)

- Systembus

- Schreib-, Lese- und Quersummentest der Speicher

- Abarbeiten von Testroutinen in der Zentraleinheit (Phantomregelkreis)

- Laufzeit- und Betriebsüberwachungssignale

Die gestörten Betriebszustände werden auf den LED's der Prozeßinterface-Komponenten angezeigt, zusätzlich erfolgt ein Ausdruck auf dem Drucker.

Selbsttätige Reaktionen des Contronic P-Leitsystems:

- Übergang auf „Inselbetrieb“ der Pozeßstationen

- Datenrettungsroutinen in der Zentralleitstation bei Netzausfall

- Ereignismeldung 


\section{c) Bussystem}

"Leistungsdaten: Übertragung:

Anzahl der Stationen: max. 127 (1-adressig), davon max. 32 aktiv Buslänge:

Struktur:

Kapazität:

'Die passiven Stationen PS belasten den Bus nicht, da sie keine Kommunikation mit anderen Busteilnehmern betreiben. Folgende Funktionen der aktiven Stationen haben einen Transferbedarf auf den Systembus:

- Ereigniskoordination

- Aktualisierung der Bildinhalte

- Weiterleitung von Bedienereignissen

- Kurvenerfassung

- PS-Kommunikation

- Protokollfunktion

Die geschätzte Busauslastung liegt ca. bei $12-15 \%$ der Gesamtkapazität.

\section{Erweiterungsmöglichkeiten des Contronic P-Leitsystems}

Das PLS bietet im Rahmen weiterer Ausbaustufen des Prozesses eine vollkommene homogene Integration, sowohl für die dazu notwendige Hardware als auch für ggfs. notwendig werdende Zusatzfunktionen.

\subsection{Beschreibung des Rechnernetzes}

Das Contronic P-Prozeßleitsystem ist über eine V.-24Schnittstelle (Conlink) mit einem Hostrechner in der Betriebsleitebene verbunden. Als Hostrechner ist eine UNIX-Workstation Typ Apollo 715/50 mit dem Betriebssystem UNIX verfuigbar. Die Workstation besitzt 2 interne 1,05 Gbyte Festplattenspeicher. An die Workstation sind ein Monitor mit Tastatur, ein Laserdrucker, ein HPGLDrucker DIN A 3, ein Matrixdrucker DIN A 3 und ein Bandlaufwerk 3,6 Gbyte angeschlossen. Über eine Ethernet-Schnittstelle sind 5 PC's Marke HP Vektra VL 2 mit der Workstation vernetzt. Die Kommunikationssoftware TCPIP verbindet sowohl die 5 PC's mit der Workstation als auch die PC's untereinander. Die PC's haben darüberhinaus eine VT 340-Terminal Emulation. Durch die Vernetzung besteht ein beliebiger Zugriff zwischen den PC's, der Workstation, Peripheriegeräten und Contronic P. Auf der UNIX Workstation ist das Betriebsleitebenenprogramm $\mathrm{CIM} / 21$ installiert. Es handelt sich dabei um eine Echtzeitplattform zur Visualisierung, Archivierung, Protokollierung, Verarbeitung und Verteilung von Prozeßdaten. Das seitens der TFH Wildau beigestellte Simulationsprogramm Aspen Plus ist auf der UNIX-Workstation installiert. Die Ankopplung der Prozeßdaten zum Aspen Plus wird über ASCII-Files realisiert. Das Programm Chemcad ist als DOS/Windows-Anwendung über die Terminals direkt verfügbar.

\section{Prozeßmodellierung - Stand der Technik und Entwicklung}

Die klassische Verfahrensentwicklung vom Labor über experimentelle Pilotierung und Scale up bis zum endgültigen Verfahren ist langwierig und aufwendig und deshalb gerade in Deutschland in immer weniger Anwendungsfällen wirtschaftlich vertretbar. Demgegenüber lassen sich viele Untersuchungen, die heute beispielsweise in den zentralen Technika der deutschen Großchemie noch experimentell durchgeführt werden, rechnerisch schneller, preiswerter und bequemer durchführen. Dabei entwickelt sich EDV-Prozeßmodellierung immer mehr zu einem unentbehrlichen Werkzeug des forschenden, entwickelnden und apparatebauenden Verfahrensingenieurs.

Seit etwa dreißig Jahren prinzipiell bekannt, ist der industrielle Bedarf an Software für Apparateauslegung, Fließschema-Berechnung, Prozeßmodellierung und -simulation von (nordamerikanischen) Softwareanbietern bereits in den siebziger Jahren erkannt worden. Seither fehit es nicht an unterschiedlich gut geeigneten, kommerziell erwerbbaren Softwareprogrammen, die sich vor allem in der stationären Flowsheet-Simulation, z. B. im Apparate- und Anlagenbau, bewährt haben. Diese Programme ermöglichen - unterschiedlich komfortabel - die Berechnung von Massen- und Energiebilanzen eines Fließbildes, der darin enthaltenen Hauptapparate, zugehöriger Ein- und Ausgangsgrößen, wie Mengenströme, Temperatur, Druck und Stoffdaten. Gefragt sind Programme, die vom Verfahrenstechniker ohne zusätzlichen Programmieraufwand unmittelbar für seine individuelle Fragestellung verwendbar sind. Zur Zeit werden z. B. ASPEN CHEMCAD oder HYSIM von vielen größeren Chemie- und Anlagenbaufirmen genutzt. Auch an nicht wenigen deutschen Hochschulen haben einige dieser Programme Einzug gehalten. Für kleine und mittlere Firmen wäre Hilfe aus dem Hochschulbereich bei der Auswahl des geeigneten Rechenprogrammes willkommen. Aus der Sicht kleinerer Firmen kann es z. B. interessant sein, bei Bedarf Berechnungen auf dem Wege der Kooperation, z. B. in einem Hochschulinstitut, durchführen zu lassen und sich somit Anschaffungs- und Unterhaltungskosten für ein aufwendiges Rechenprogramm ganz zu sparen.

Während sich demnach die stationäre Prozeßmodellierung als kostengünstiges Werkzeug des Verfahrensingenieurs etabliert hat, die praktischen Schwierigkeiten haupsächlich in der Auswahl des geeigneten Programmpaketes, allenfalls noch in der Ausbildung und im Bereithalten von qualifiziertem Personal bestehen, hat die dynamische Prozeß- und Anlagensimulation - etwa bei nichtstationärer Prozeßfuihrung von An- und Abfahrvorgängen, Batchverfahren usw. - in der industriellen Praxis noch keine breite Anwendung gefunden. Intensive Untersuchungen zum Einsatz universell einsetzbarer Simulatoren werden erst seit Anfang der achziger Jahre durchgeführt. Die Problematik ist wegen der zusätzlichen Echtzeit-Fragestellung wesentlich komplexer, der Zeitaufwand ungleich größer und der damit verbundene Kostenaufwand derzeit nur in Sonderfällen vertretbar. Hier besteht noch erheblicher Forschungs- und Entwicklungsbedarf auch an den Hochschulen. 


\section{Kabelplan}

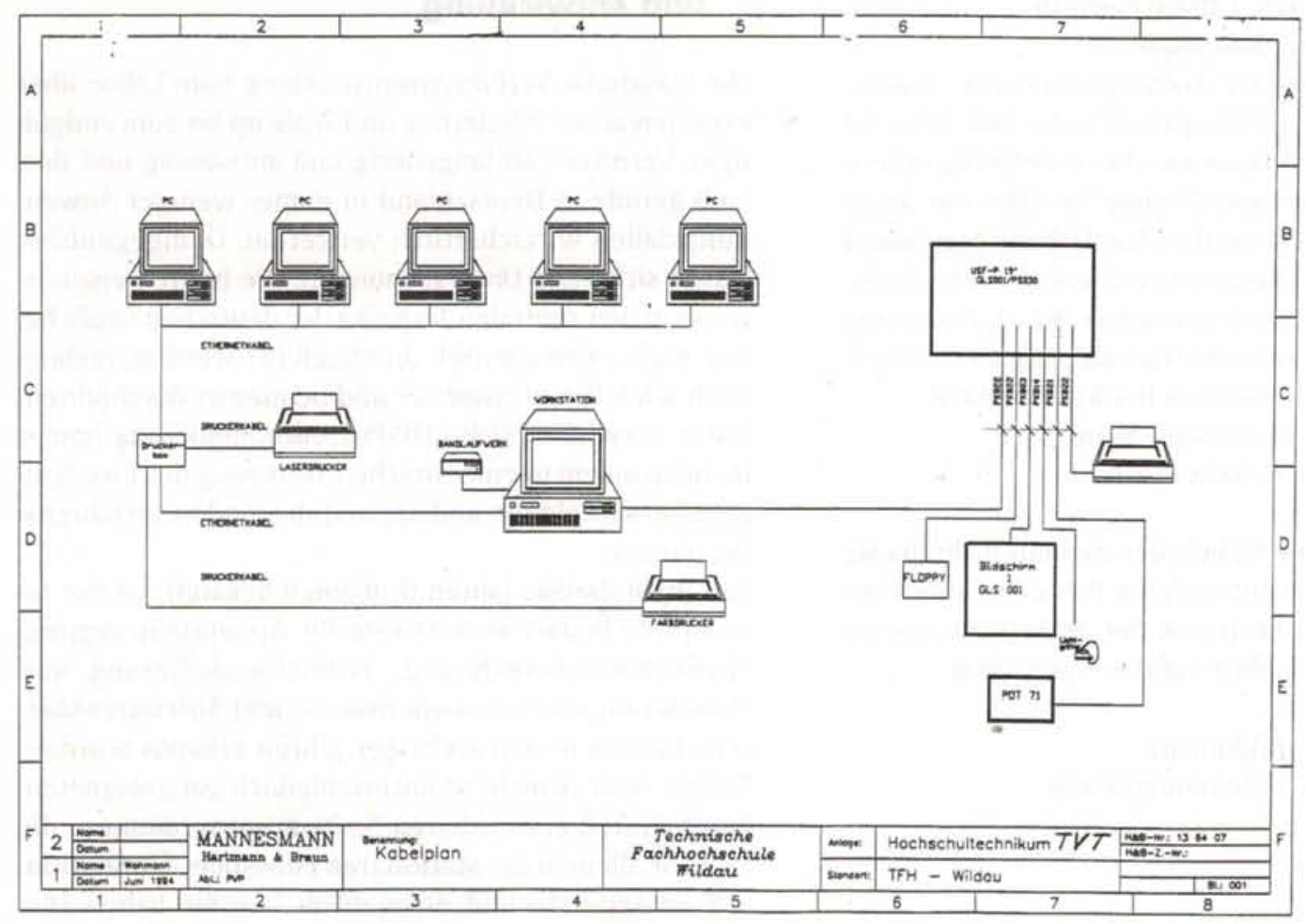

Hardwarekonfiguration

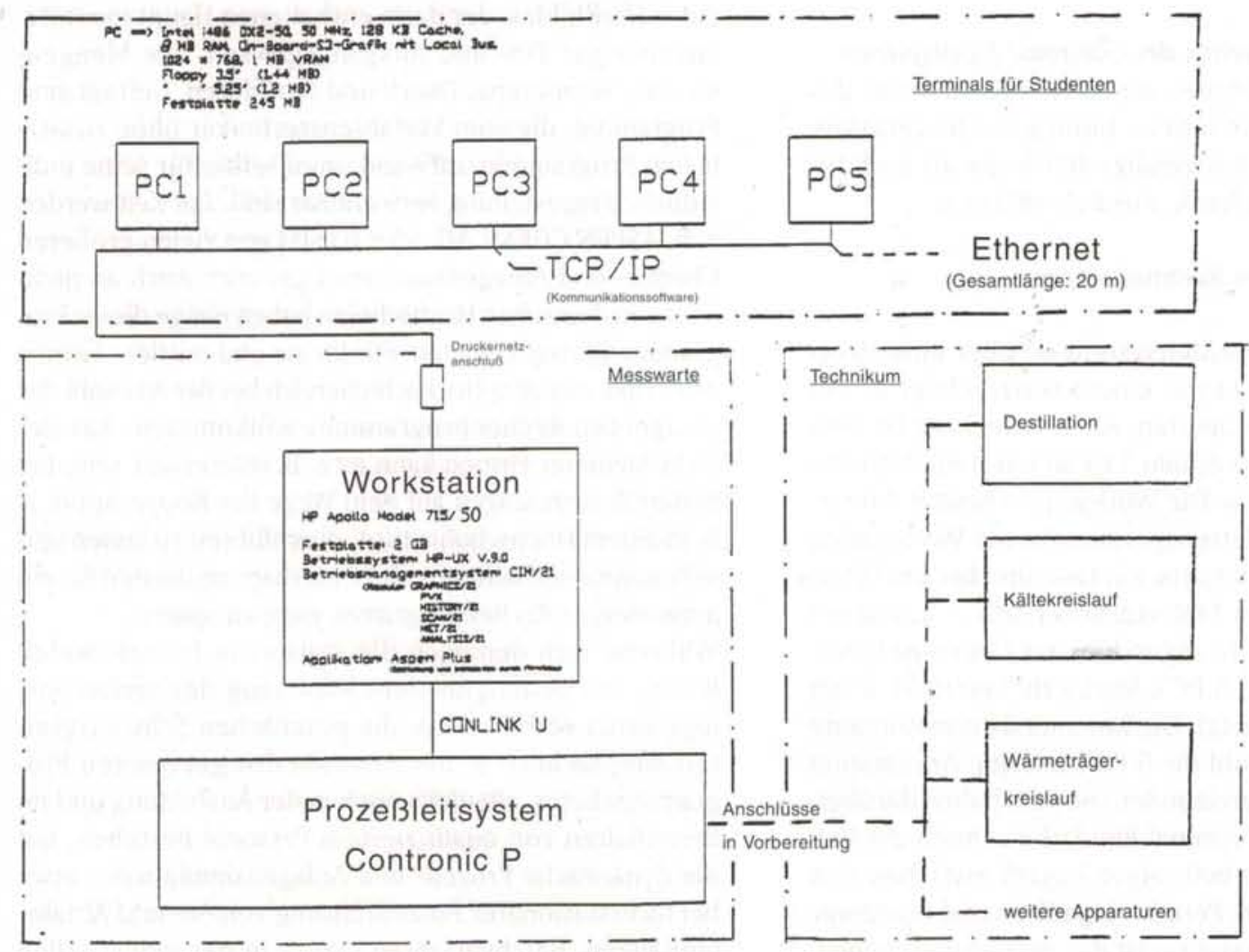

\section{Literatur}

[1] Marquardt, W. et al.: Prozeßsimulation, VCH Verlagsgesellschaft mbH Weinheim usw., 1995

[2] Williams, S.: Process Modelling With [DMO], Real Time Optimisation Of Ethylene Plants, Chemical Technology Europe, 1 (5), 34-36, 1994

[3] Ingham, J., Dunn, I. J.: Desktop Simulation Of Dynamic Chemical Engineering Processes, 1 (5), 14-20, 1994

\section{Verfasser}

Prof. Dr.-Ing. Josef Schmadl Technische Fachhochschule Wildau Fachbereich Verfahrenstechnik Tel. (0 33 75) 507-110 